\title{
A Note on the Numerical Solution of the Wave Equation With Piecewise Smooth Coefficients
}

\author{
By David L. Brown
}

\begin{abstract}
The numerical solution of the initial value problem for the wave equation is considered for the case when the equation coefficients are piecewise smooth. This problem models linear wave propagation in a medium in which the properties of the medium change discontinuously at interfaces. Convergent difference approximations can be found that do not require the explicit specification of the boundary conditions at interfaces in the medium and hence are simple to program. Although such difference approximations typically can only be expected to be first-order accurate, the numerical phase velocity has the same accuracy as the difference approximation would if the coefficients in the differential equation were smoooth. This is proved for the one-dimensional case and demonstrated numerically for an example in two space dimensions in which the interface is not aligned with the computational mesh.
\end{abstract}

1. Introduction. In this note we consider the numerical solution by finite difference approximation of the scalar wave equation

$$
\rho(x, y) \frac{\partial^{2} u}{\partial t^{2}}-\nabla \cdot \mu(x, y) \nabla u=0
$$

on $-\infty<x, y<\infty, t \geqslant 0$ with initial conditions $u(x, y, 0)$ and $\partial u(x, y, 0) / \partial t$ specified. Here $u=u(x, y, t)$ is a scalar function of its arguments and $\rho(x, y)$, $\mu(x, y)$ are piecewise smooth coefficients. This problem models linear wave propagation in a piecewise smooth medium. Efficient and accurate methods for solving such problems numerically are of interest in the modelling of seismic wave propagation in geophysics [1], [4].

Suppose for the moment that $\rho$ and $\mu$ are piecewise constant. For definiteness we assume that $-\infty<x, y<\infty$ is divided up into two semi-infinite regions by the curve $f(x, y)=0$, and that $\rho=\rho_{1}, \mu=\mu_{1}$ for $f(x, y)<0$ and $\rho=\rho_{2}, \mu=\mu_{2}$ for $f(x, y)>0$. Because of the discontinuity in the coefficients along $f(x, y)=0$, additional conditions on the dependent variable $u$ must be specified in order to uniquely determine the solution of (1.1). The usual conditions are that $u(x, y)$ and $\mu(x, y)(\partial u / \partial n)$ be continuous across the line $f(x, y)=0$. (Here $\partial u / \partial n$ is the normal derivative of $u$ on $f$.) The entire problem can be reformulated as follows:

$$
\begin{array}{ll}
\rho_{1} u_{t t}-\mu_{1} \nabla^{2} u=0 & \text { for } f(x, y)<0, \\
\rho_{2} u_{t t}-\mu_{2} \nabla^{2} u=0 & \text { for } f(x, y)>0,
\end{array}
$$

Received May 10, 1982.

1980 Mathematics Subject Classification. Primary 65M10, 65M15, 65N10, 65N15.

${ }^{*}$ Research partially supported by Office of Naval Research Contract no. N0014-80-C0076. Computer time provided by the Stanford Exploration Project, Stanford University Department of Geophysics and on the Caltech Applied Mathematics Department "Fluid Dynamics VAX". 


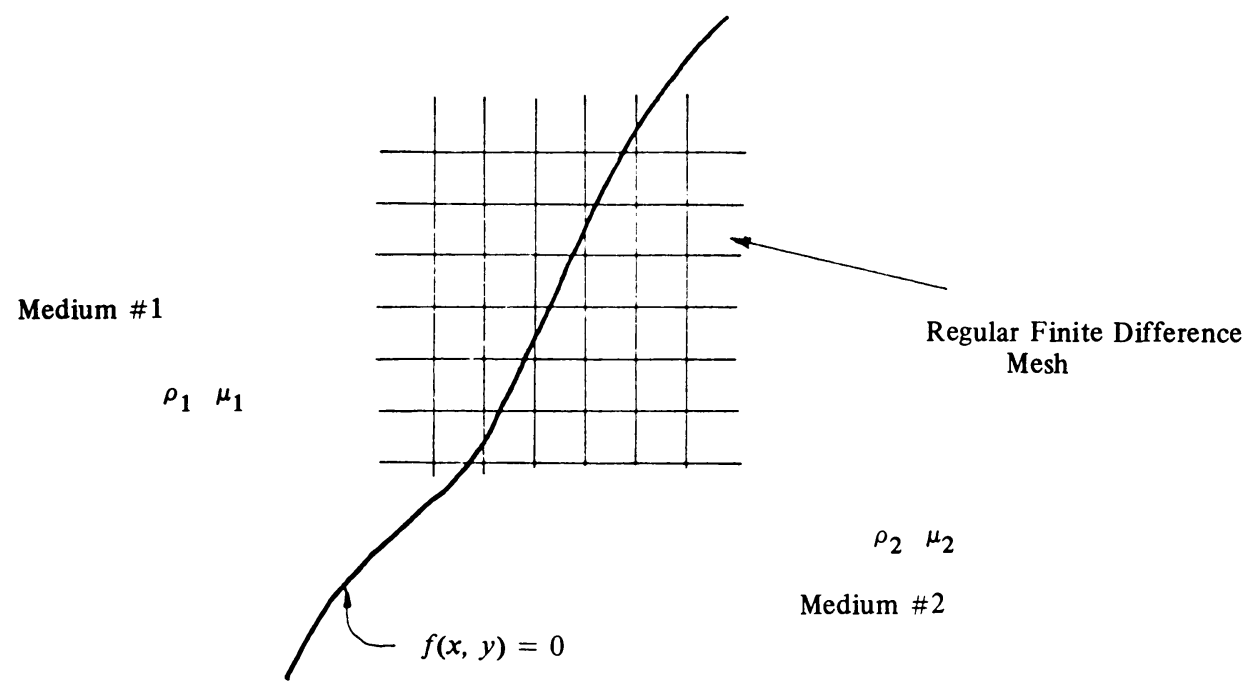

FIGURE 1. A piecewise constant medium

with

$$
\begin{gathered}
{[u(x, y)]_{f(x, y)=0}=0,} \\
{\left[\mu u_{n}(x, y)\right]_{f(x, y)=0}=0}
\end{gathered}
$$

(plus the same initial conditions as for (1.1)). Here $[g(x, y)]_{f(x, y)=0}$ is the jump in $g$ across the line $f(x, y)=0$ and subscripts denote partial differentiation. In general we are interested in the numerical solution of (1.2), (1.3) for arbitrary smooth curves $f(x, y)=0$. If a rectangular finite difference mesh is used, the (approximate) specification of the interface conditions (1.3) can be difficult since the curve $f(x, y)=0$ may not be aligned with that mesh.

The purpose of this note is to point out two simple results on difference approximations for (1.2), (1.3) that can be helpful in the situation just described. Although these results follow from well-known results for finite difference approximations to hyperbolic equations, they are apparently not well understood. It is (in principal) straightforward to find finite difference approximations to the problem (1.1) that are of arbitrary order of accuracy (say $p$ ) when the coefficients $\mu$ and $\rho$ are smooth functions. The first result of interest is that such a difference approximation can be used for the problem (1.2), (1.3) with piecewise constant coefficients and will converge to the true solution of that problem in the limit of meshwidth going to zero. In particular, the method will typically be a $p$ th-order accurate approximation to the differential equation (1.2) and at least a first-order accurate approximation to the interface conditions (1.3). (The same result holds for the corresponding piecewisesmooth coefficient problem as well.)

For each frequency component of a computed solution to the problem (1.2), (1.3), the error can be decomposed into a phase velocity error and an amplitude error that is possibly complex but constant as a function of location $(x, y)$. The second result of interest is that if a centered difference approximation is used to approximate the differential equation (1.2), the accuracy with which the phase velocity is computed is the same as the accuracy with which the differential equation (1.2) is approximated, 
while the accuracy with which the amplitude is computed is determined by the accuracy with which the interface conditions (1.3) are approximated. (This result assumes, of course, that an exact representation of the initial data is used.)

Suppose that the initial data for the problem (1.2), (1.3) consist of a wave pulse located somewhere to the left of the interface $f(x, y)=0$ and moving initially towards the interface. In the exact solution to the problem, the pulse moves towards the interface until it reaches it. An interaction with the interface occurs, and reflected and transmitted wave pulses result. In the numerical solution to (1.2), (1.3), essentially the same phenomena are observed, but, due to the phase error of the solution, the wave pulse disperses and will propagate with incorrect group velocity both before and after it interacts with the interface. (This is well known and is discussed, for example, by Trefethen [6]). As a result, after some time the location of the pulse can be entirely incorrect. On the other hand, the amplitude of the reflected and transmittted pulses is determined only by the approximation to the interface condition (1.3) and so does not deteriorate in accuracy once the pulse has interacted with the interface. One can argue, therefore, that it is much more important to use a high-order approximation to the differential equation (1.2) than it is to use a high-order approximation to the interface condition (1.3). An implication of the two results stated above is, therefore, that an adequate numerical approximation to the problem (1.2), (1.3) can be obtained without explicitly approximating the interface conditions (1.3). This is a very important conclusion from the point-of-view of minimizing the complexity of a computer program which is to be used for modelling linear wave propagation in a piecewise-smooth medium.

2. Decomposition of the Computational Error. The computation error associated with a difference approximation to the problem (1.2), (1.3) can be decomposed into an amplitude error and a phase velocity error. In this section we will show that if the difference method used to approximate the differential equation (1.2) (the "interior approximation") is centered, then the phase velocity error results entirely from this interior approximation, while the error in the amplitude results from the inaccuracies associated with the approximation of the interface conditions (1.3). This result is actually fairly obvious as we can show by the following explicit computation.

To simplify the comparison with the solution of the difference approximation we choose to solve (1.2), (1.3) in one space dimension and by using a Laplace transform over $t$. The problem can be restated as follows:

$$
\begin{array}{cl}
u_{t t}-c_{1}^{2} u_{x x}=0 & \text { for }-\infty<x \leqslant 0, t \geqslant 0, \\
v_{t t}-c_{2}^{2} v_{x x}=0 & \text { for } 0 \leqslant x<\infty, t \geqslant 0,
\end{array}
$$

with interface conditions

$$
u(0, t)=v(0, t), \quad c_{1}^{2} u_{x}(0, t)=c_{2}^{2} v_{x}(0, t)
$$

and initial conditions

$$
u(x, 0)=f(x), \quad u_{t}(x, 0)=-c f^{\prime}(x) \text { for }-\infty<x<\infty,
$$

where $f(x) \in C_{0}^{\infty}(-\infty<x \leqslant \delta)$ for some $\delta<0$. (Although not explicitly mentioned below, we take $\delta<-n h$ in order that Eq. (2.16a) be valid. $n$ and $h$ are defined below.) Here, for convenience, we have taken $\rho_{1}=\rho_{2} \equiv 1$ and represented $\mu_{1}, \mu_{2}$ as 
$c_{1}^{2}, c_{2}^{2}$, the square of the velocities in each medium. The initial conditions can be thought of as a wave pulse moving initially to the right, for example.

To solve the problem (2.1)-(2.3) we Laplace transform (2.1) over $t$ and use (2.3) to obtain for each $s$ with $\operatorname{Re} s \geqslant 0$ the ordinary differential equations

$$
\hat{u}_{x x}-\frac{s^{2}}{c_{1}^{2}} \hat{u}=\frac{1}{2 \pi}\left(\frac{1}{c_{1}} f^{\prime}(x)-\frac{s}{c_{1}^{2}} f(x)\right)
$$

and

$$
\hat{v}_{x x}-\frac{s^{2}}{c_{2}^{2}} \hat{v}=0
$$

where

$$
\hat{w}=\hat{w}(x, s):=\frac{1}{2 \pi} \int_{0}^{\infty} w(x, t) e^{-s t} d t
$$

defines the Laplace transform of the function $w(x, t)$. The interface conditions (2.2) become

$$
\hat{u}(0, s)=\hat{v}(0, s) \quad \text { and } \quad c_{1}^{2} \hat{u}_{x}(0, s)=c_{2}^{2} \hat{v}_{x}(0, s) .
$$

It is clear by substitution that a particular solution of the inhomogeneous equation $(2.4 \mathrm{a})$ is given by

$$
\hat{U}(x, s)=\frac{1}{2 \pi} \int_{0}^{\infty} e^{-s t} f\left(x-c_{1} t\right) d t .
$$

The general solution (bounded for all $\operatorname{Re} s \geqslant 0)$ of $(2.4)$ is then given by

$$
\hat{u}(x, s)=\hat{U}(x, s)+\sigma_{1}(s) e^{s x / c_{1}}, \quad \hat{v}(x, s)=\sigma_{2}(s) e^{-s x / c_{2}},
$$

where the exponential functions $e^{s x / c_{1}}$ and $e^{-s x / c_{2}}$ are fundamental solutions of the homogeneous forms of (2.4a) and (2.4b), respectively, and $\sigma_{1}, \sigma_{2}$ are coefficients that will be determined by the interface conditions. Substitution of (2.7) into the interface conditions (2.2) gives for $\sigma_{1}, \sigma_{2}$ the values

$$
\sigma_{1}(s)=\frac{c_{1}-c_{2}}{c_{1}+c_{2}} \hat{U}(0, s), \quad \sigma_{2}(s)=\frac{2 c_{1}}{c_{1}+c_{2}} \hat{U}(0, s) .
$$

Substitution of (2.8) into (2.7) follows by inverse Laplace transformation yields the following representation for the solution to (2.1)-(2.3) in terms of Fourier transforms:

$$
\begin{gathered}
u(x, t)=f\left(x-c_{1} t\right)+\frac{1}{2 \pi} \int_{-\infty}^{\infty} \frac{c_{1}-c_{2}}{c_{1}+c_{2}} \hat{U}(0, i \omega) e^{i \omega\left(t+x / c_{1}\right)} d \omega, \\
v(x, t)=\frac{1}{2 \pi} \int_{-\infty}^{\infty} \frac{2 c_{1}}{c_{1}+c_{2}} \hat{U}(0, i \omega) e^{i \omega\left(t-x / c_{2}\right)} d \omega .
\end{gathered}
$$

An interpretation of this solution is the following: The initial pulse $f(x)$ moves to the right with speed $c_{1}$ until it reaches the boundary. (This part of the solution depends only on the differential equation and the intial data.) At the boundary, it is partially reflected and partially transmitted. The reflection and transmission coefficients are given by $R=\left(c_{1}-c_{2}\right) /\left(c_{1}+c_{2}\right)$ and $T=2 c_{1} /\left(c_{1}+c_{2}\right)$, respectively, and were determined by the interface conditions. Each frequency component 
$\hat{U}(0, i \omega)$ of the reflected wave moves to the left with speed $-c_{1}$. Similarly, each frequency component of the transmitted wave moves to the right with speed $c_{2}$. (This is clear from looking at the phase of the complex exponentials in the integrals. Since those complex exponentials were the fundamental solutions of the differential equations, it is again obvious that the propagation of the reflected and transmitted waves is determined only by the differential equation.)

In the rest of this section we will demonstrate that the solution of a centered difference approximation to the problem (2.1)-(2.3) behaves in the same way, i.e., the phase (and its error) are determined by the interior approximation and the reflection and transmission coefficients (and their errors) are determined by the interface approximation.

We approximate (2.1) with a time-continuous finite difference approximation given by

$$
\begin{gathered}
\frac{\partial^{2} u_{\nu}}{\partial t^{2}}-c_{1}^{2} Q(E) u_{\nu}=0, \quad \nu=-n,-n-1,-n-2, \ldots, \\
\frac{\partial^{2} v_{\nu}}{\partial t^{2}}-c_{2}^{2} Q(E) v_{\nu}=0, \quad \nu=n+1, n+2, \ldots
\end{gathered}
$$

where

$$
Q(E):=\frac{1}{h^{2}} \sum_{j=0}^{n} \beta_{j}\left(E^{j}+E^{-j}\right)
$$

is a centered difference operator of width $2 n+1$ and consistent with $\partial^{2} / \partial x^{2}$. Here $u_{\nu}=u_{\nu}(t)$ and $v_{\nu}=v_{\nu}(t)$ are approximations to $u\left(x_{\nu}, t\right)$ and $v\left(x_{\nu}, t\right)$, respectively. $E w_{\nu}:=w_{\nu+1}$, and the meshpoints $x_{\nu}$ are defined by $x_{\nu}=\nu h+\gamma$ where $h<\gamma<h$. (The uniform meshwidth is given by $h$.) The interface conditions (2.2) are approximated with the $2 n$ relations given by

$$
B_{1}^{(\mu)}(E) u_{0}(t)=B_{2}^{(\mu)}(E) v_{0}(t), \quad \mu=1,2, \ldots, 2 n .
$$

For the understanding of the error, it is not important to specify the difference operators $B_{1}^{(\mu)}$ and $B_{2}^{(\mu)}$ in detail, although it is clear that we must require that the relations (2.11) be consistent with the interface conditions (2.2). The initial data for the problem (2.10), (2.11) are taken as

$$
u_{\nu}(0)=f\left(x_{\nu}\right) \quad \frac{\partial}{\partial t} u_{\nu}(0)=-c f^{\prime}\left(x_{\nu}\right) .
$$

As in the continuous case, we will solve the discrete problem (2.10)-(2.12) explicitly using Laplace transforms. After Laplace transformation, the problem is replaced with, for each $s$ with $\operatorname{Re} s \geqslant 0$, the ordinary difference equations

$$
\begin{gathered}
Q(E) \hat{u}_{\nu}-\frac{s^{2}}{c_{1}^{2}} \hat{u}_{\nu}=\frac{1}{2 \pi}\left(\frac{1}{c_{1}} f^{\prime}(x)-\frac{s}{c_{1}^{2}} f(x)\right), \\
Q(E) \hat{u}_{\nu}-\frac{s^{2}}{c_{2}^{2}} \hat{u}_{\nu}=0,
\end{gathered}
$$


with interface conditions

$$
B_{1}^{(\mu)}(E) \hat{u}_{0}(t)=B_{2}^{(\mu)}(E) \hat{v}_{0}(t), \quad \mu=1,2, \ldots, 2 n .
$$

Since $(2.13 \mathrm{~b})$ is a homogeneous difference equation with constant coefficients, its general solution is given by linear combinations of powers of the roots $\kappa_{j}(s)$, $j=1,2, \ldots, 2 n$ of the characteristic equation

$$
\kappa^{n}\left(Q(\kappa)-\frac{s^{2}}{c_{2}^{2}}\right)=0
$$

The solution of the homogeneous form of (2.13a) is determined in a similar way, with corresponding characteristic roots denoted by $\lambda_{j}(s), j=1,2, \ldots, 2 n$. It is well known (see,e.g., Gustafsson, Kreiss and Sundström [3, Section 5]) that for $\operatorname{Re} s>0$ the roots $\kappa_{j}(s)$ separate into two distinct groups: $M_{1}(\kappa)$ containing those roots $\kappa_{j}(s)$ with $\left|\kappa_{j}(s)\right|<1$ and $M_{2}(\kappa)$ containing those roots $\kappa_{j}(s)$ with $\left|\kappa_{j}(s)\right|>1$. The number of roots in each group, counted according to their multiplicity is independent of $s$ for $\operatorname{Re} s>0$. Furthermore, since (2.13b) is a centered difference approximation, each of $M_{1}(\kappa), M_{2}(\kappa)$ contains exactly $n$ roots. Exactly the same result is true for $M_{1}(\lambda)$ and $M_{2}(\lambda)$. Hence, corresponding to Eqs. (2.7) for the continuous problem, the general solution (bounded for all $s$ with $\operatorname{Re} s \geqslant 0$ ) of (2.13) is given by

$$
\begin{gathered}
\hat{u}_{\nu}(s)=\hat{U}_{\nu}(s)+\sum_{\lambda_{j} \in M_{2}(\lambda)} P_{j}(\nu) \lambda_{j}(s)^{\nu} \text { for } \nu \leqslant 0, \\
\hat{v}_{\nu}(s)=\sum_{\kappa_{j} \in M_{1}(\kappa)} \tilde{P}_{j}(\nu) \kappa_{j}(s)^{\nu} \text { for } \nu \geqslant 0,
\end{gathered}
$$

where $\hat{U}_{\nu}(s)$ is a particular solution of $(2.13 \mathrm{a})$ and $P_{j}(\nu), \tilde{P}_{j}(\nu)$ are polynomials in $\nu$ of degree equal to the multiplicity of $\lambda_{j}$, respectively, $\kappa_{j}$ minus one. Since $M_{2}\left(\lambda_{j}\right)$ contains $n$ roots, the coefficients in Eq.(2.16a) depend on $n$ free parameters $r_{1}, \ldots, r_{n}$. Similarly the coefficients in (2.16b) depend on $n$ free parameters $r_{n+1}, \ldots, r_{2 n}$. These parameters are determined by substituting Eqs. (2.16) into the interface conditions (2.14), whence we obtain a $2 n \times 2 n$ linear system of equations

$$
D(s) \mathbf{r}=\hat{U}_{\nu}(s) \mathbf{b},
$$

where $\mathbf{r}:=\left(r_{1}, r_{2}, \ldots, r_{2 n}\right)^{T}, \mathbf{b}$ is a vector of length $2 n$ and $D(s)$ is a $2 n \times 2 n$ matrix. The system (2.17) can be solved boundedly for $\mathbf{r}$ if the discrete problem (2.10)-(2.12) is stable. (Compare with Lemma 10.3 of Gustafsson, Kreiss and Sundström [3].)

The representation of the solution of (2.10)-(2.12) in terms of Laplace transforms can be written down by using the following lemmas, which can be taken as obvious:

LEMMA 1. If the difference approximation (2.10) is accurate of order $p$, then one of the $\lambda_{j} \in M_{2}(\lambda)$ can be written as

$$
\lambda_{1}(s)=e^{s h\left(1+O\left(s^{p} h^{p}\right)\right) / c_{1}} .
$$

Similarly one of the $\kappa_{j} \in M_{1}(\kappa)$ can be expressed as

$$
\kappa_{1}(s)=e^{-s h\left(1+O\left(s^{p} h^{p}\right)\right) / c_{2}} .
$$

Furthermore, $\lambda_{1}$ and $\kappa_{1}$ are simple roots and $\left|\lambda_{1}(i \omega)\right|=\mid \kappa_{1}(i \omega) \equiv 1$. 
LEMMA 2. If (2.10) is accurate of order p, then the particular solution of (2.13a) can be expressed as

$$
\hat{U}_{\nu}(s)=\hat{U}\left(x_{\nu}, s\right)\left(1+O\left(s^{p} h^{p}\right)\right) .
$$

LEMMA 3. If the interface approximation is accurate of order $q$, then the solution of (2.17) gives

$$
\begin{aligned}
& P_{1}(\nu)=\frac{c_{1}-c_{2}}{c_{1}+c_{2}} \hat{U}_{0}(s)\left(1+O\left(s^{q} h^{q}\right)\right), \\
& \tilde{P}_{1}(\nu)=\frac{2 c_{1}}{c_{1}+c_{2}} \hat{U}_{0}(s)\left(1+O\left(s^{q} h^{q}\right)\right),
\end{aligned}
$$

and $P_{j}(\nu)=O\left(s^{q} h^{q}\right), \tilde{P}_{j}(\nu)=O\left(s^{q} h^{q}\right)$ for $j \neq 1$.

It is obvious, therefore, that corresponding to (2.9) we have the following representation for the solution of the discrete problem $(2.10)-(2.12)$ in terms of Fourier transforms:

$$
\begin{aligned}
& u_{\nu}(t)=\frac{1}{2 \pi} \int_{-\infty}^{\infty} e^{i \omega t} \hat{U}_{\nu}(i \omega) d \omega \\
& +\frac{1}{2 \pi} \int_{-\infty}^{\infty}\left\{\frac{c_{1}-c_{2}}{c_{1}+c_{2}}\left(1+O\left(\omega^{q} h^{q}\right)\right) \hat{U}(0, i \omega)\right\} \\
& \quad \times e^{i \omega\left(t+x\left(1+O\left(\omega^{p} h^{p}\right)\right) / c_{1}\right)} d \omega, \\
& \begin{aligned}
u_{\nu}(t)=\frac{1}{2 \pi} \int_{-\infty}^{\infty}\left\{\frac{2 c_{1}}{c_{1}+c_{2}}\left(1+O\left(\omega^{q} h^{q}\right)\right) \hat{U}(O, i \omega)\right\} \\
\times e^{i \omega\left(t-x\left(1+O\left(\omega^{p} h^{p}\right)\right) / c_{2}\right)} d \omega .
\end{aligned}
\end{aligned}
$$

(Here we have assumed that $q \leqslant p$.) The second integral in Eq. (2.21a) represents the reflected wave. Equation (2.21b) gives the transmitted wave. Comparing the Eqs. (2.9), we see that the reflection and transmission coefficients $\tilde{R}$ and $\tilde{T}$ associated with the difference approximation are related to the true coefficients by

$$
\tilde{R}(\omega h)=R\left(1+O\left(\omega^{q} h^{q}\right)\right) \text { and } \tilde{T}(\omega h)=T\left(1+O\left(\omega^{q} h^{q}\right)\right) .
$$

Again, each frequency component of the reflected and transmitted wave moves into its respective medium with (frequency dependent) speed

$$
-\tilde{c}_{1}(\omega h)=-c_{1}\left(1+O\left(\omega^{p} h^{p}\right)\right) \quad \text { and } \quad \tilde{c}_{2}(\omega h)=c_{2}\left(1+O\left(\omega^{p} h^{p}\right)\right),
$$

respectively. Note also that since the difference approximation is centered, the complex exponential in each of the integrals in Eqs. (2.21) has unit magnitude, i.e. there is no decay of amplitude in the waves as they propagate. We have therefore proved

THEOREM (DECOMPOSITION OF THE ERROR). If the difference aproximation (2.10) is accurate of order $p$, the interface approximation (2.11) is accurate of order $q \leqslant p$, and the method (2.10), (2.11) is stable, then the reflection and transmission coefficients $\tilde{R}(\omega h), \tilde{T}(\omega h)$ associated with the interface will be accurate of order $q$ while the phase velocities $\tilde{c}_{1}, \tilde{c}_{2}$ of the discrete media will be accurate of order $p$. 
3. Convergence and Numerical Examples. In this section we explicitly calculate reflection and transmission coefficients for a difference approximation to the problem (2.1)-(2.3). We also give numerical evidence to illustrate the conclusions of the introduction. For convenience we will actually consider a similar problem given by

$$
u_{t t}=a^{2} u_{x x} \text { on }-1 \leqslant x \leqslant 0
$$

and

$$
v_{t t}=b^{2} v_{x x} \text { on } 0 \leqslant x<\infty
$$

with interface conditions

$$
u(0, t)=v(0, t), \quad a^{2} u_{x}(0, t)=b^{2} v_{x}(0, t) .
$$

For the purposes of this example we will consider a boundary value problem in which a signal propagates into the region $[-1, \infty)$ from the left. For this reason boundary conditions

$$
u(-1, t)=f(t)
$$

are given. Furthermore we specify homogeneous initial conditions:

$$
\begin{aligned}
& u(x, 0)=u_{t}(x, 0) \equiv 0, \quad-1 \leqslant x \leqslant 0, \\
& v(x, 0)=v_{t}(x, 0) \equiv 0, \quad 0 \leqslant x<\infty \text {. }
\end{aligned}
$$

We now Laplace transform the problem over $t$ and obtain for each frequency $s$ with $\operatorname{Re} s \geqslant 0$ a boundary value problem for the ordinary differential equation

$$
\begin{aligned}
s^{2} \hat{u} & =a^{2} \hat{u}_{x x}, & & -1 \leqslant x \leqslant 0, \\
s^{2} \hat{v} & =b^{2} \hat{v}_{x x}, & & 0 \leqslant x<\infty .
\end{aligned}
$$

The interface and boundary conditions become

$$
\hat{u}(-1, s)=\hat{f}(s), \quad \hat{u}(0, s)=\hat{v}(0, s), \quad a^{2} \hat{u}_{x}(0, s)=b^{2} \hat{v}_{x}(0, s) .
$$

The problem (3.5)-(3.6) has bounded solutions of the form

$$
\begin{gathered}
\hat{u}(x, s)=\sigma_{1} e^{-s x / a}+\sigma_{2} e^{s x / a}, \quad-1 \leqslant x<0, \\
\hat{v}(x, s)=\sigma_{3} e^{-s x / b}, \quad 0 \leqslant x<\infty,
\end{gathered}
$$

for all $s$ with $\operatorname{Re} s \geqslant 0$. Substituting the general solution (3.7) and (3.8) into the boundary conditions (3.6), we obtain a linear system of equations for the constants $\sigma_{i}, i=1,2,3$. Solving this system, we can find the reflection and transmission coefficients, $R$ and $T$ respectively, which are given by

$$
R:=\frac{\sigma_{2}}{\sigma_{1}}=\frac{a-b}{a+b}, \quad T:=\frac{\sigma_{3}}{\sigma_{1}}=\frac{2 a}{a+b} .
$$

(This is, of course, the same result we found in Section 3.)

We now approximate the problem (3.1)-(3.3) with the time-continuous difference approximation given by

$$
\frac{\partial^{2} w_{\nu}}{\partial t^{2}}=D_{+} c_{\nu}^{2} D_{-} w_{\nu}, \quad \nu=-N, \ldots,-1,0,1, \ldots
$$


with boundary conditions $w_{-N}(t)=f(t)$ and initial conditions $w_{\nu}(0)=\partial w_{\nu}(0) / \partial t \equiv$ 0 . Here $D_{ \pm} w_{\nu}:=h^{-1} \Delta_{ \pm} w_{\nu}, w_{\nu}(t)=w\left(x_{\nu}, t\right)$ is an approximation to $u\left(x_{\nu}, t\right)$ for $x<0$ and to $v\left(x_{\nu}, t\right)$ for $x>0$. The meshpoints are given by $x_{\nu}=(\nu-\alpha) h$ where $h=(N+\alpha)^{-1}$. (See diagram below.)

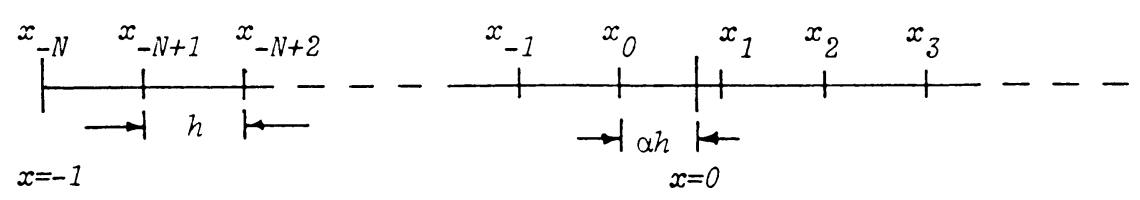

Intuitively, we expect problem (3.10) to give a second-order approximation to the differential equation (3.1) and at least a first-order approximation to the interface conditions (3.2) if $c_{\nu}$ is a consistent representation of the velocity function

$$
c(x)= \begin{cases}a & \text { for } x<0 \\ b & \text { for } x>0\end{cases}
$$

In this section we are particularly interested in considering the following choice for $c_{\nu}$ which gives a second-order approximation to the interface conditions. This representation was proposed by Tikhonov and Samarskiu [5] for second-order ordinary differential equations with discontinuous coefficients:

$$
c_{\nu}^{2}= \begin{cases}a^{2} & \text { for } \nu \leqslant 0 \\ \left(\alpha / a^{2}+(1-\alpha) / b^{2}\right)^{-1} & \text { for } \nu=1 \\ b^{2} & \text { for } \nu \geqslant 2 .\end{cases}
$$

(In general for the differential equation with variable coefficients $u_{t t}=\left(a^{2}(x) u_{x}\right)_{x}$, $c_{\nu}$ is given by $c_{\nu}^{-2}=\int_{(\nu-1) h}^{\nu h} a(x)^{-2} d x$.) The problem (3.10), (3.11) can be solved

\begin{tabular}{|c|c|c|c|c|c|}
\hline \multicolumn{6}{|c|}{ Discrete $L_{2}$-norm errors at $t=1.5$} \\
\hline $\begin{array}{c}\text { Method } \\
\text { Interface Location } \\
\end{array}$ & $\begin{array}{l}\text { Secc } \\
h \\
\end{array}$ & $\begin{array}{l}\text { 1d-order } \\
x<0 \\
\end{array}$ & $\begin{array}{l}(3.15) \\
x>0 \\
\end{array}$ & $\begin{array}{l}\text { Fourth } \\
x<0 \\
\end{array}$ & $\begin{array}{l}\operatorname{der}(3.16) \\
x>0 \\
\end{array}$ \\
\hline$\alpha=0.0$ & $\begin{array}{l}1 / 10 \\
1 / 20 \\
1 / 40 \\
1 / 80\end{array}$ & $\begin{array}{r}1.0102 \\
.5415 \\
.1442 \\
.0365\end{array}$ & $\begin{array}{l}.6569 \\
.3433 \\
.1033 \\
.0257\end{array}$ & $\begin{array}{l}.2777 \\
.0656 \\
.0040 \\
.0004\end{array}$ & $\begin{array}{l}.1191 \\
.0261 \\
.0025 \\
.0004\end{array}$ \\
\hline$\alpha=0.2$ & $\begin{array}{l}1 / 10 \\
1 / 20 \\
1 / 40 \\
1 / 80\end{array}$ & $\begin{array}{r}1.0201 \\
.4858 \\
.1329 \\
.0351\end{array}$ & $\begin{array}{l}.6381 \\
.3471 \\
.1034 \\
.0257\end{array}$ & $\begin{array}{l}.1564 \\
.0412 \\
.0047 \\
.0010\end{array}$ & $\begin{array}{l}.1431 \\
.0296 \\
.0026 \\
.0004\end{array}$ \\
\hline$\alpha=0.5$ & $\begin{array}{l}1 / 10 \\
1 / 20 \\
1 / 40 \\
1 / 80\end{array}$ & $\begin{array}{r}1.04 .49 \\
.4928 \\
.1363 \\
.0358\end{array}$ & $\begin{array}{l}.6331 \\
.3534 \\
.1042 \\
.0257\end{array}$ & $\begin{array}{l}.2635 \\
.0665 \\
.0129 \\
.0031\end{array}$ & $\begin{array}{l}.1355 \\
.0371 \\
.0037 \\
.0007\end{array}$ \\
\hline
\end{tabular}

TABLE 1 
explicitly using Laplace transforms in a similar manner as for the continuous problem above. Note that, in this case, the (Laplace-transformed) "interface conditions" are taken as

$$
s^{2} \hat{w}_{\nu}=D_{+} c_{\nu} D_{-} \hat{w}_{\nu} \quad \text { for } \nu=0,1
$$

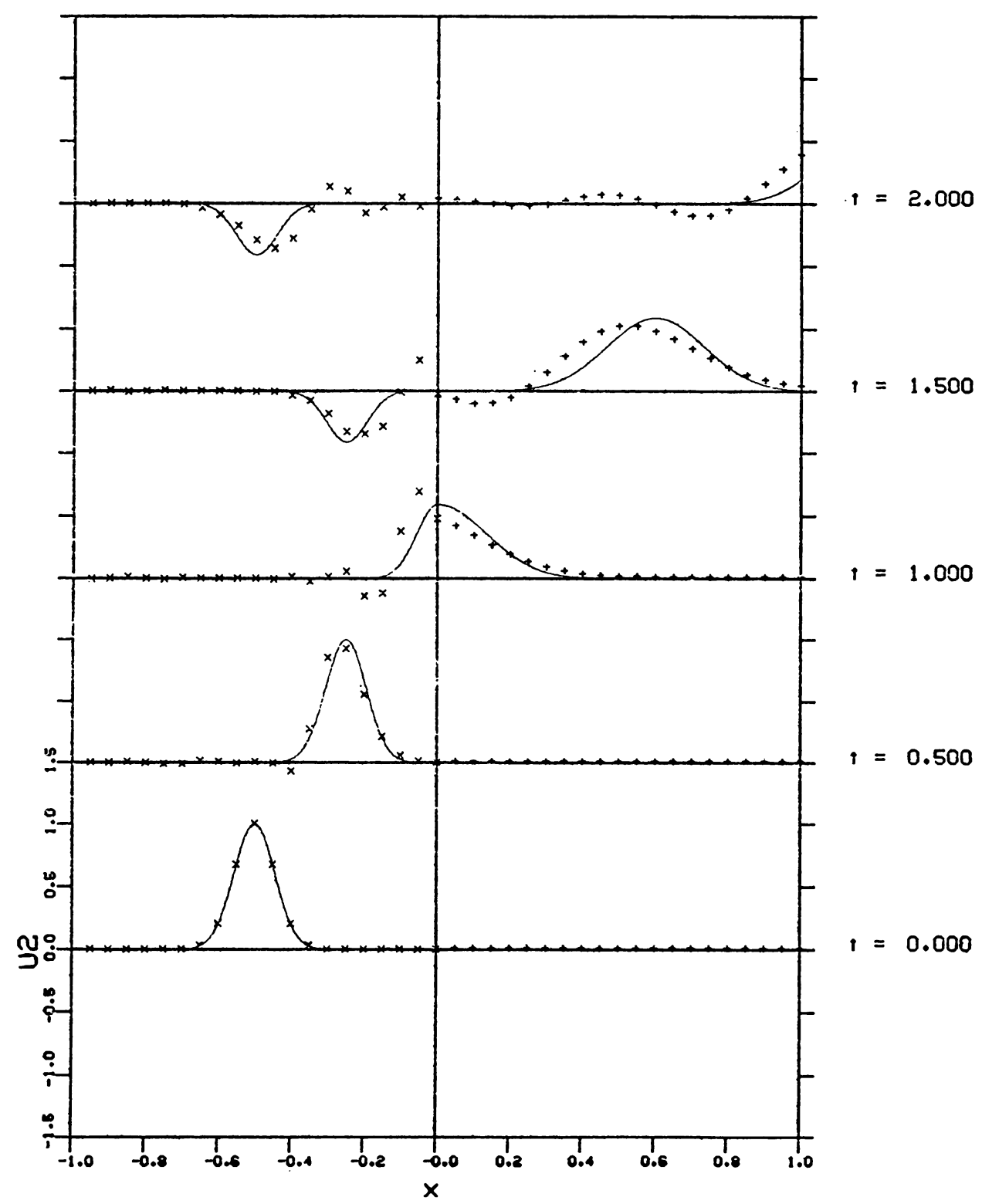

FIGURE 2. Second-order method with second-order interface approximation 
The reflection and transmission coefficients can be derived, and are second-order accurate for all choices of $0 \leqslant \alpha \leqslant 1$. In particular, for $\alpha=0$,

$$
\begin{gathered}
\tilde{R}(s h)=R\left(1+\frac{s^{2} h^{2}}{4 a b}+O\left(s^{3} h^{3}\right)\right), \\
\tilde{T}(s h)=T\left(1+\frac{b-a}{8 a^{2} b} s^{2} h^{2}+O\left(s^{3} h^{3}\right)\right) .
\end{gathered}
$$

Numerical computations were made for an interface problem for the wave equation (3.1) on $-1 \leqslant x \leqslant 1$ with the interface at $x=0$. Initial conditions were specified so

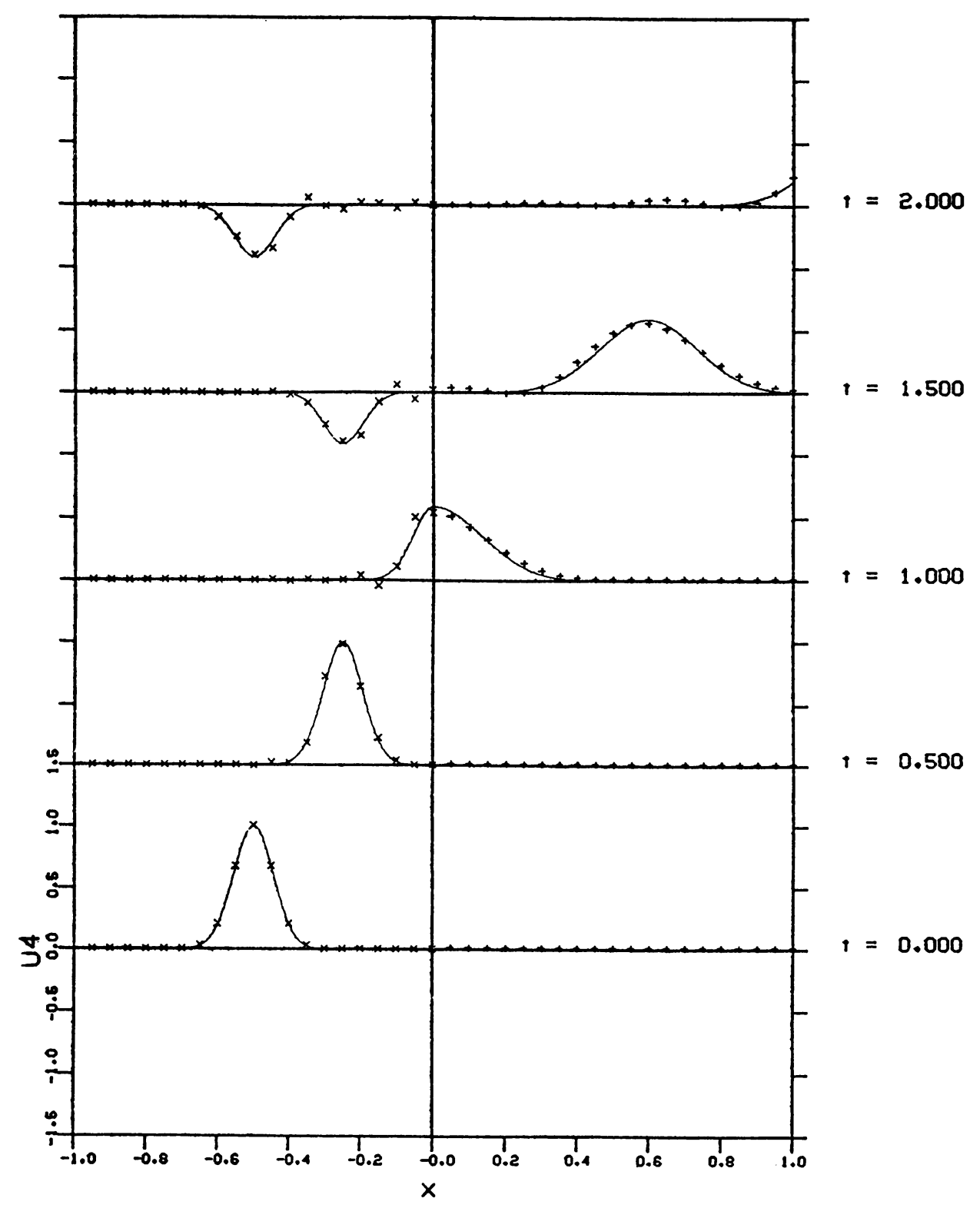

FIGURE 3. Fourth-order method with second-order interface approximation 


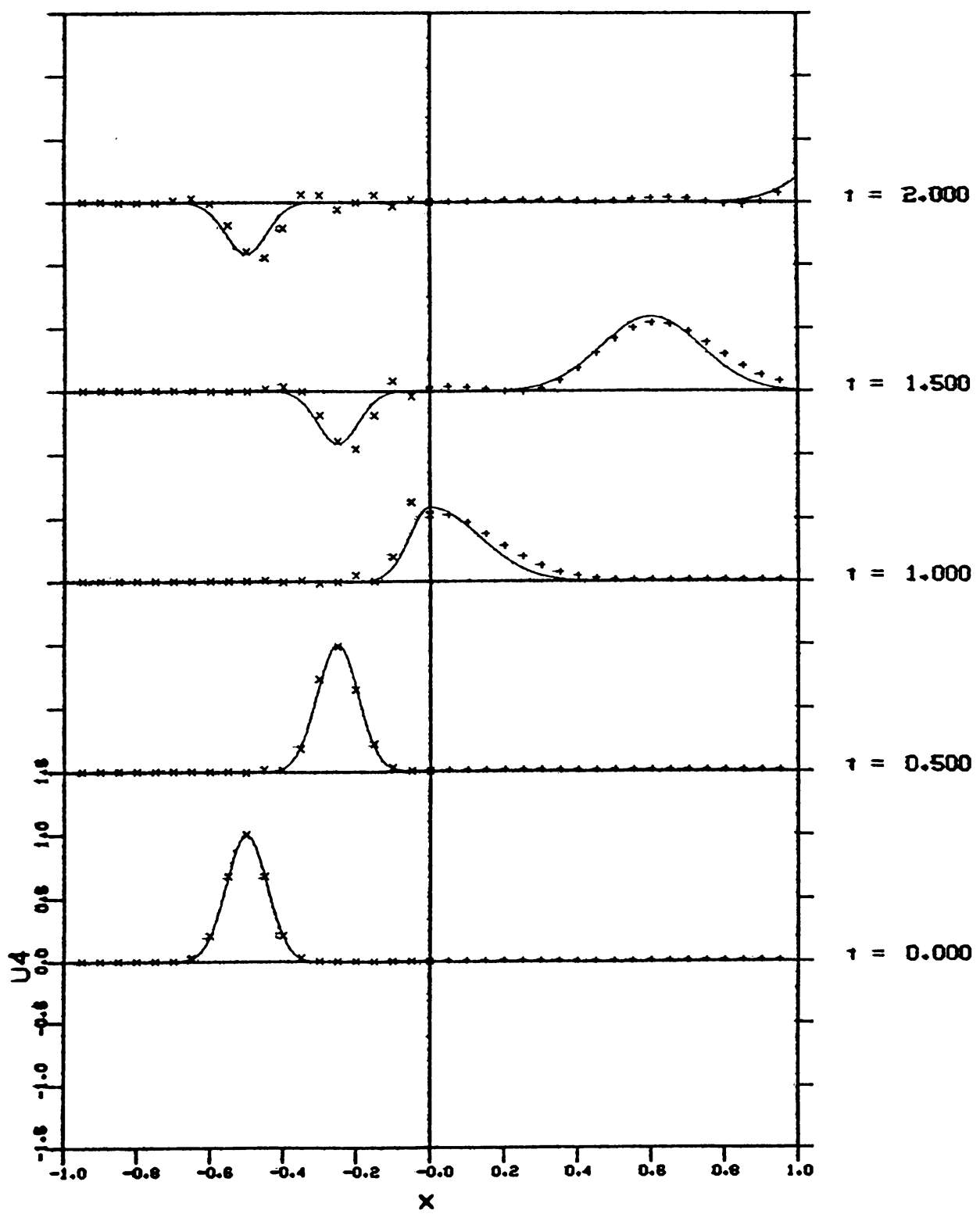

FIGURE 4. Fourth-order method with first-order interface approximation

that a pulse would propagate from the region $-1<x<0$ to the interface at $x=0$ where reflected and transmitted signals are generated. For the interior approximation, we used both a second-order method

$$
w_{\nu}(t+k)=2 w_{\nu}(t)-w_{\nu}(t-k)+k^{2} D_{+}\left(c_{\nu}^{2} D_{-} w_{\nu}(t)\right)
$$

for $\nu=1,2, \ldots, 2 N-1$, where $N h=1$ and $x_{\nu}:=-1+(\nu-\alpha) h$ and a method with fourth-order space differences,

$$
\begin{aligned}
w_{\nu}(t+k)= & 2 w_{\nu}(t)-w_{\nu}(t-k)+k^{2} D_{+} c_{\nu}^{2} D_{-} w_{\nu}(t) \\
& -\frac{k^{2} h^{2}}{24}\left(D_{+} D_{-}\left(D_{+} c_{\nu}^{2} D_{-} w_{\nu}(t)\right)+D_{+}\left(c_{\nu}^{2} D_{+} D_{-}^{2} w_{\nu}(t)\right)\right)
\end{aligned}
$$


for $\nu=2,3, \ldots, N-2$. (In the latter computations, (3.14) was used for $\nu=1$ and $\nu=2 N-1$.) We also used Richardson extrapolation in $t$ to improve the accuracy of the solutions computed with (3.15) to fourth order in $t$. The initial conditions specified were

$$
w_{\nu}(0)=\exp \left(-160\left(x_{\nu}+.5\right)^{2}\right)
$$

and

$$
w_{\nu}(-k)=\exp \left(-160\left(x_{\nu}+a k+.5\right)^{2}\right) .
$$

At the left and right boundaries, "nonreflecting" boundary conditions were used:

$$
\begin{aligned}
w_{0}(t+k) & =w_{0}(t)+k a D_{+} w_{0}(t), \\
w_{2 N}(t+k) & =w_{2 N}(t)-k b D_{-} w_{2 N}(t) .
\end{aligned}
$$

The values $a=.5, b=1.2$ and $k / h=.5$ were used for the medium velocities and mesh ratio, respectively.

To check the convergence rate of the method we made computations with meshwidths $h=1 / 10,1 / 20,1 / 40$, and $1 / 80$ and interface location parameter $\alpha=0$., .2, and .5. The discrete $L_{2}$-norm errors on both sides of the interface at $t=1.5$ are summarized in Table 1 . (Here we define the discrete $L_{2}$-norm by $\left\|u_{\nu}\right\|_{2}=\left(\sum_{\nu=0}^{N} h u_{\nu}^{2}\right)^{1 / 2}$.) For the " $O\left(h^{2}\right)$ " approximation (3.14) it is evident that the convergence rate is $O\left(h^{2}\right)$ for all three values of $\alpha$. Although for the " $O\left(h^{4}\right)$ " approximation (3.15) the convergence rates are only somewhat better than $O\left(h^{2}\right)$, note that the magnitude of the error is greatly reduced in comparison to the second-order method. This is mainly due to the improvement of signal propagation effects that we get by using a fourth-order method in the interior. This can be seen graphically in Figures 2 and 3. These figures show a time history of the solution from $t=0.0$ to $t=2.0$. Figure 2 shows the results using the second-order approximation (3.14), and Figure 3 shows the results using the fourth-order approximation (3.15). In these figures, the solid curve represents the true solution while the symbols ' $x$ ' and ' + ' represent the calculated solution to the left and to the right of the interface, respectively. The meshwidth used for this calculation was $h=1 / 20$. Note that both the location of the pulse and its apparent amplitude are better with the fourth-order method than with the second-order method.

There does not seem to be a simple extension of this method to two space dimensions that will give second-order accuracy overall. However, even if the interface conditions are only approximated to first order, the results of Section 2 indicate that one can expect to get much better qualitative results using a fourth-order difference approximation in the computations. We demonstrate this with several numerical examples. First, in Figure 4 we show a one-dimensional example where the fourth-order approximation was used in the interior of the region and a first-order interface approximation was applied. Even though the computed solution is therefore only formally first-order accurate, the results are qualitatively better than in Figure 2, where the second-order method was used for the interior approximation.

Figures 5, 6 and 7 show the results of some computations of the wave equation in two space dimensions. The example chosen models the interaction of waves from a circular source with an interface that is oriented obliquely to the computational 


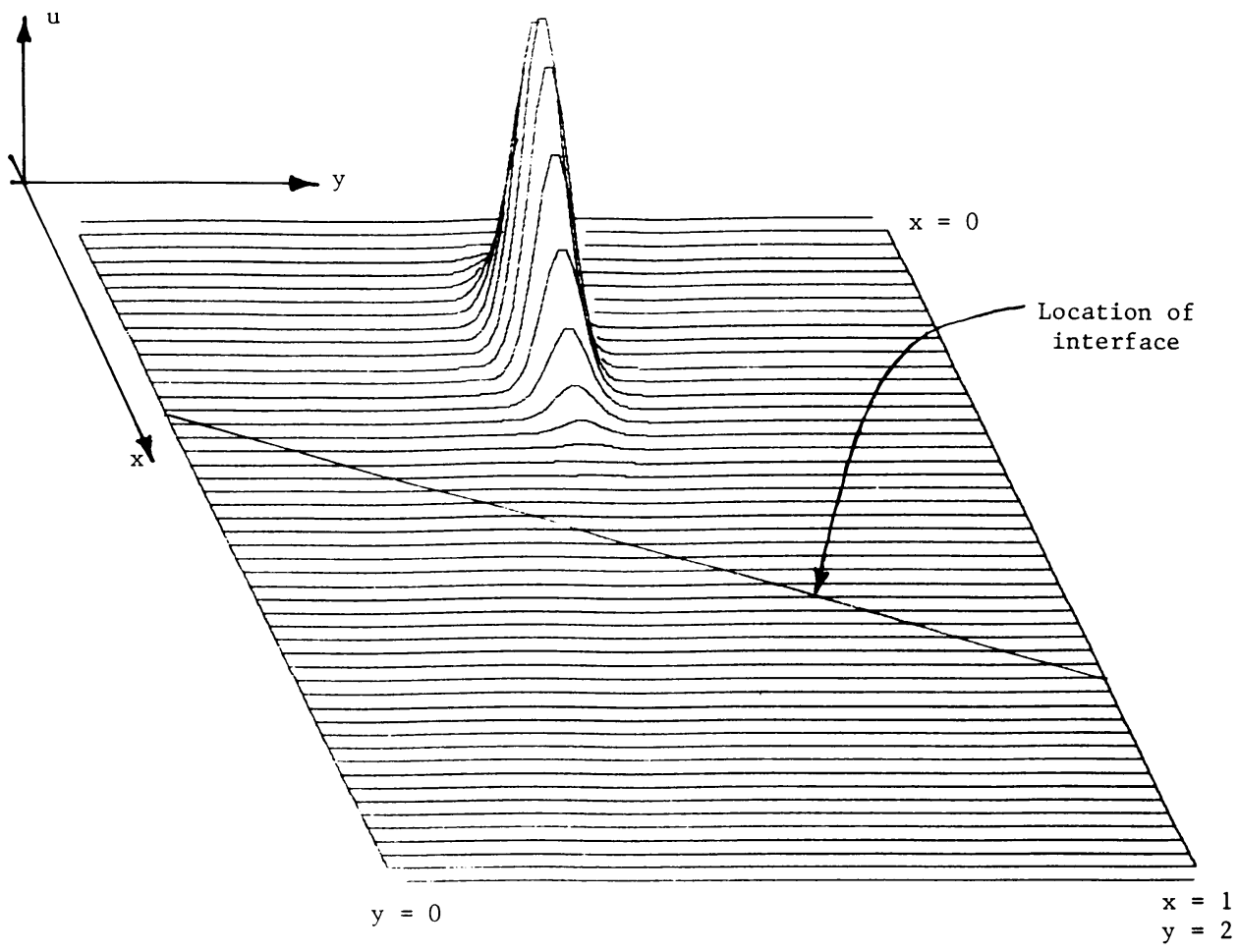

FIGURE 5a. Initial conditions for two-dimensional computation

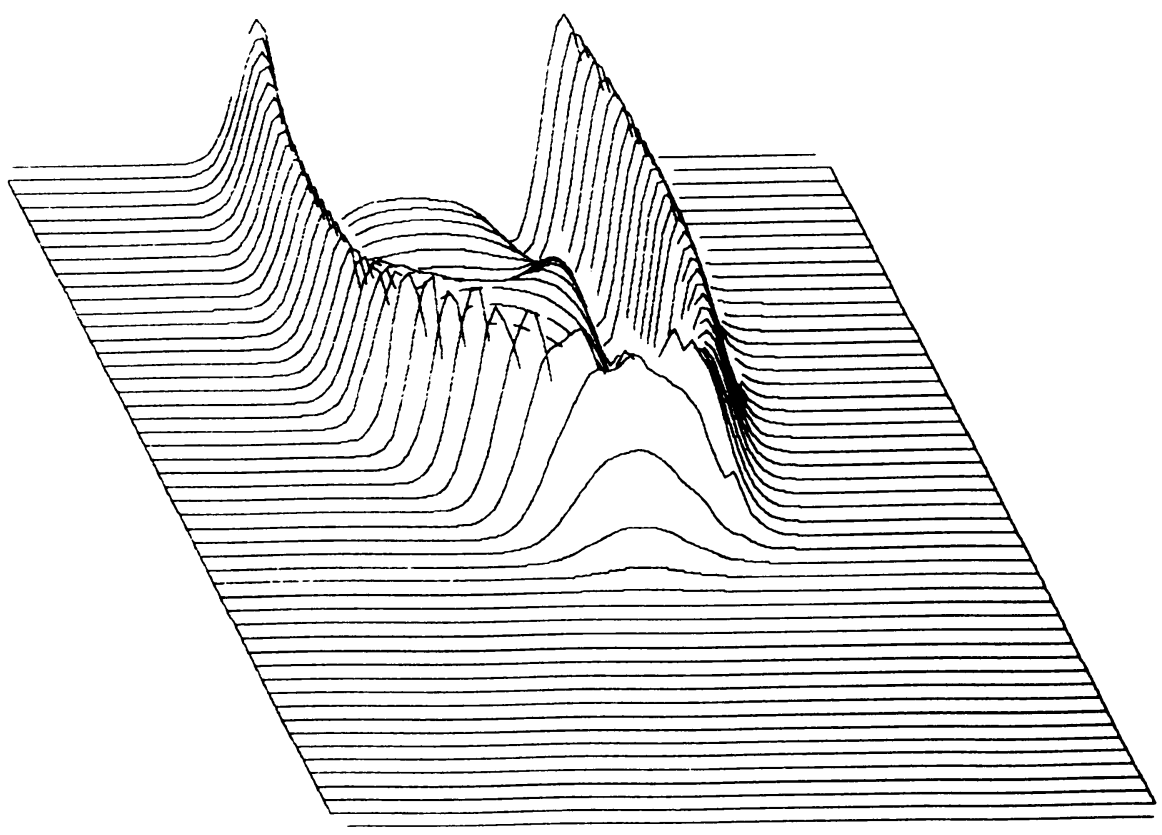

Figure 5b. Second-order method $t=.4$ 


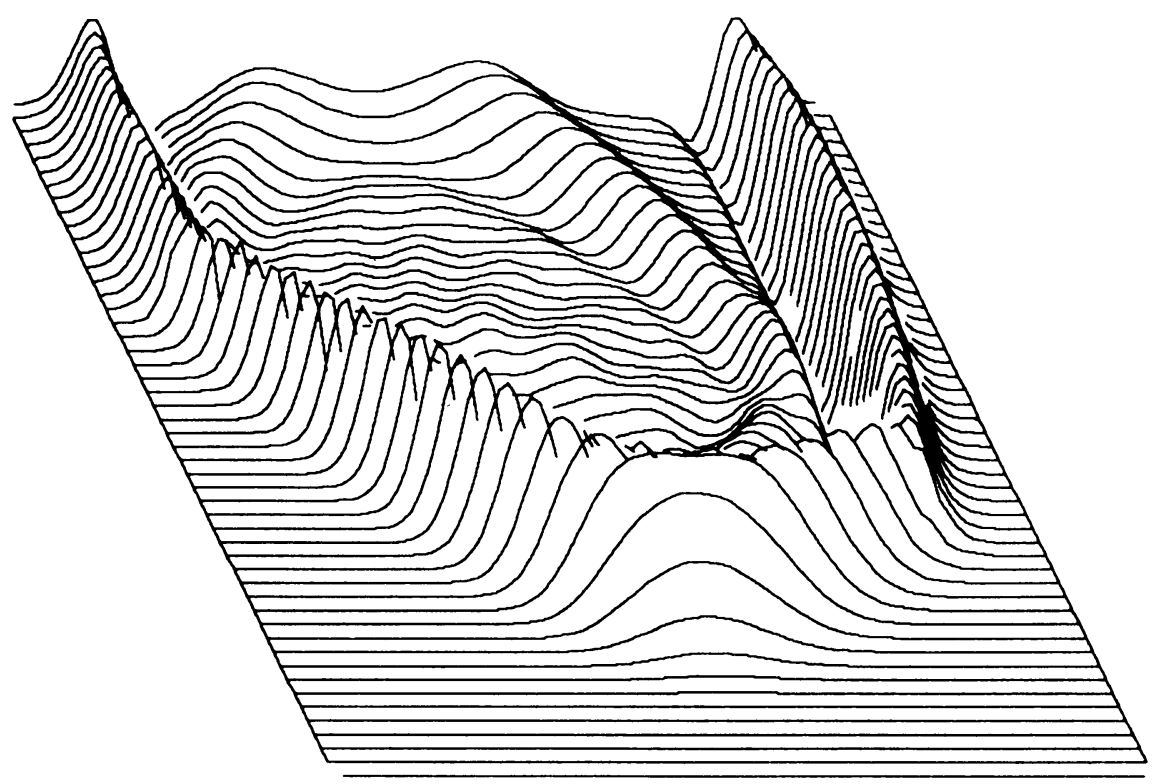

FIGURE 5c. Second-order method $t=.8$

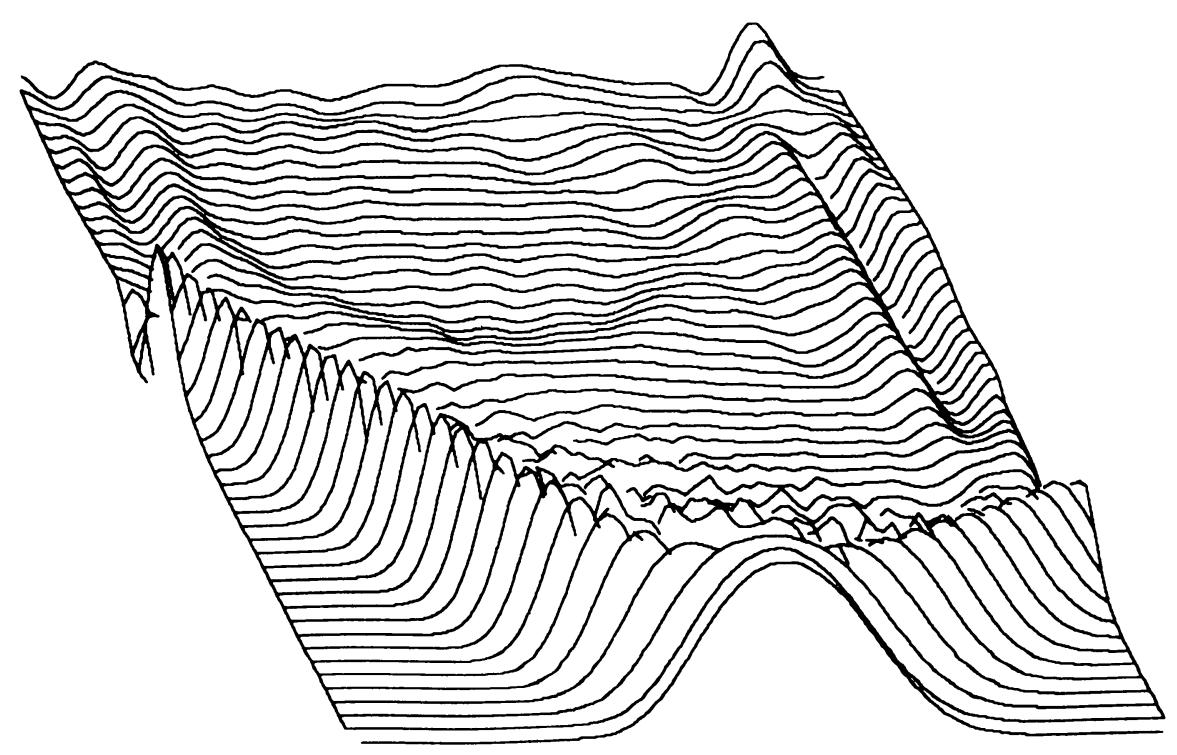

FIGURE 5d. Second-order method $t=1.2$ 


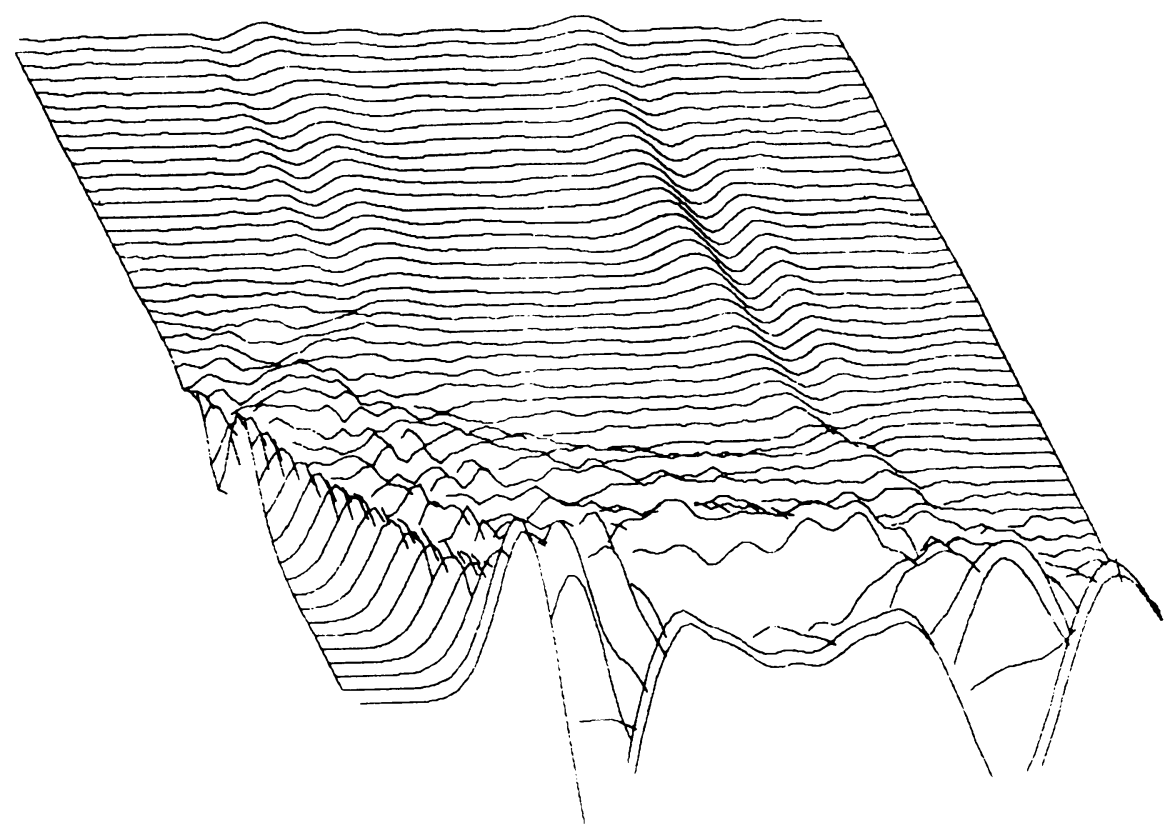

FIGURE 5e. Second-order method $t=1.6$

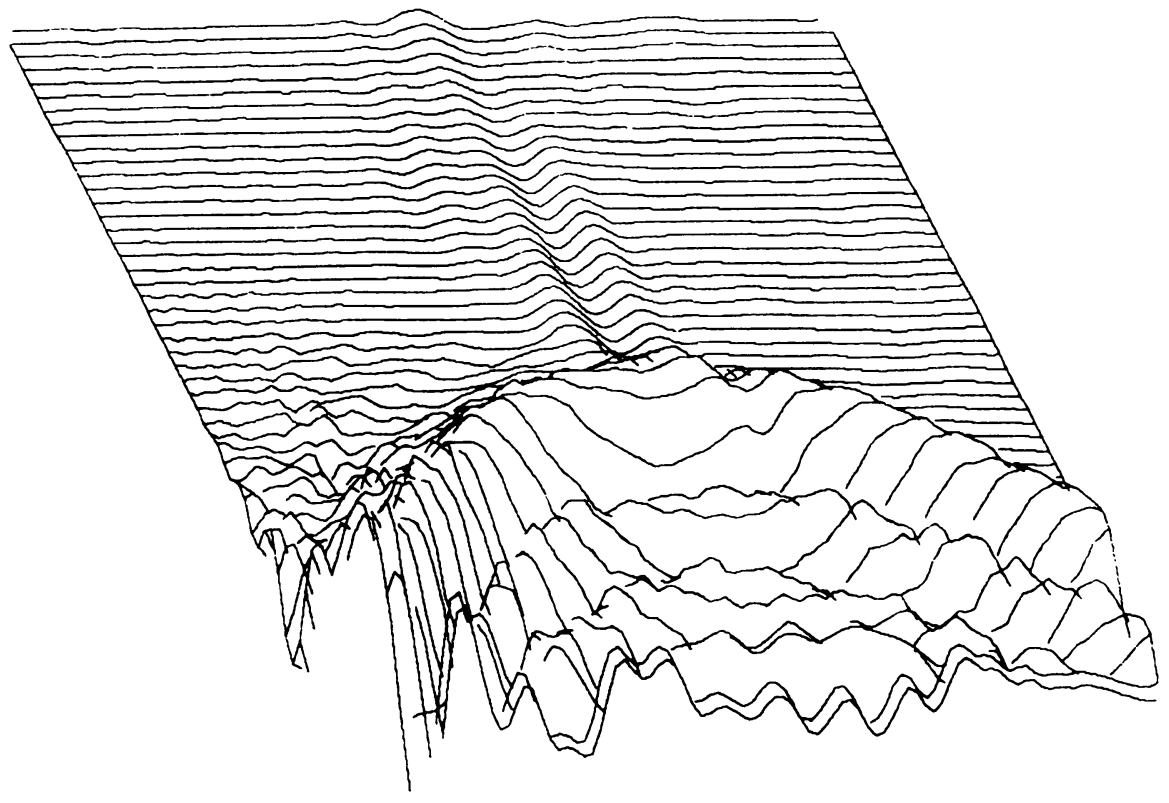

FIGURE 5f. Second-order method $t=2.0$ 


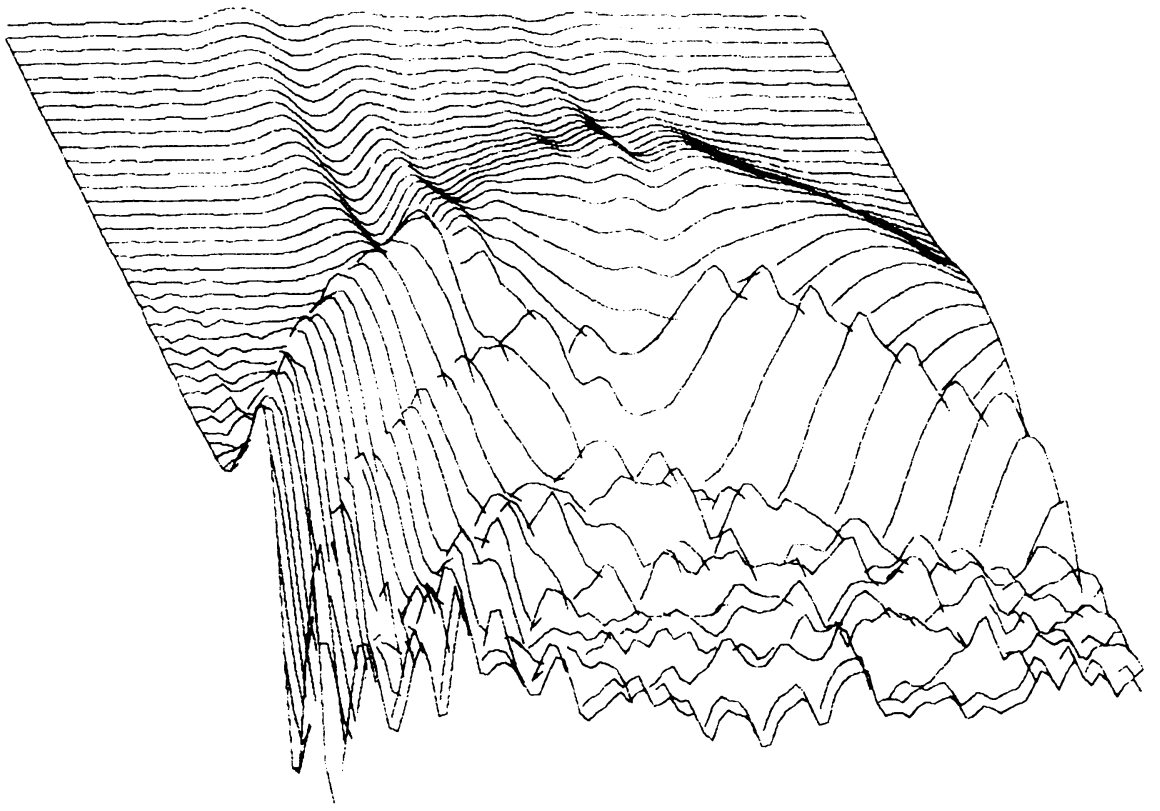

FIGURE 5g. Second-order method $t=2.4$

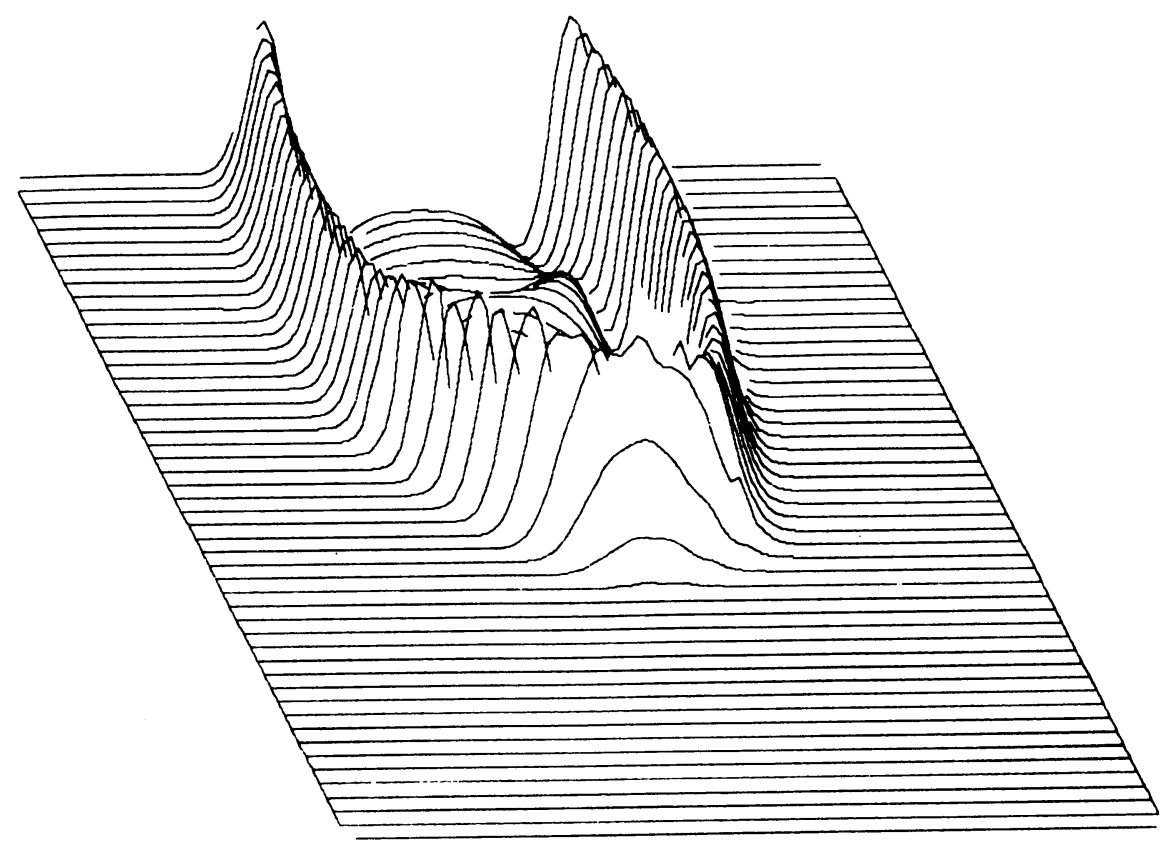

FIGURE 6a. Fourth-order method $t=.4$ 


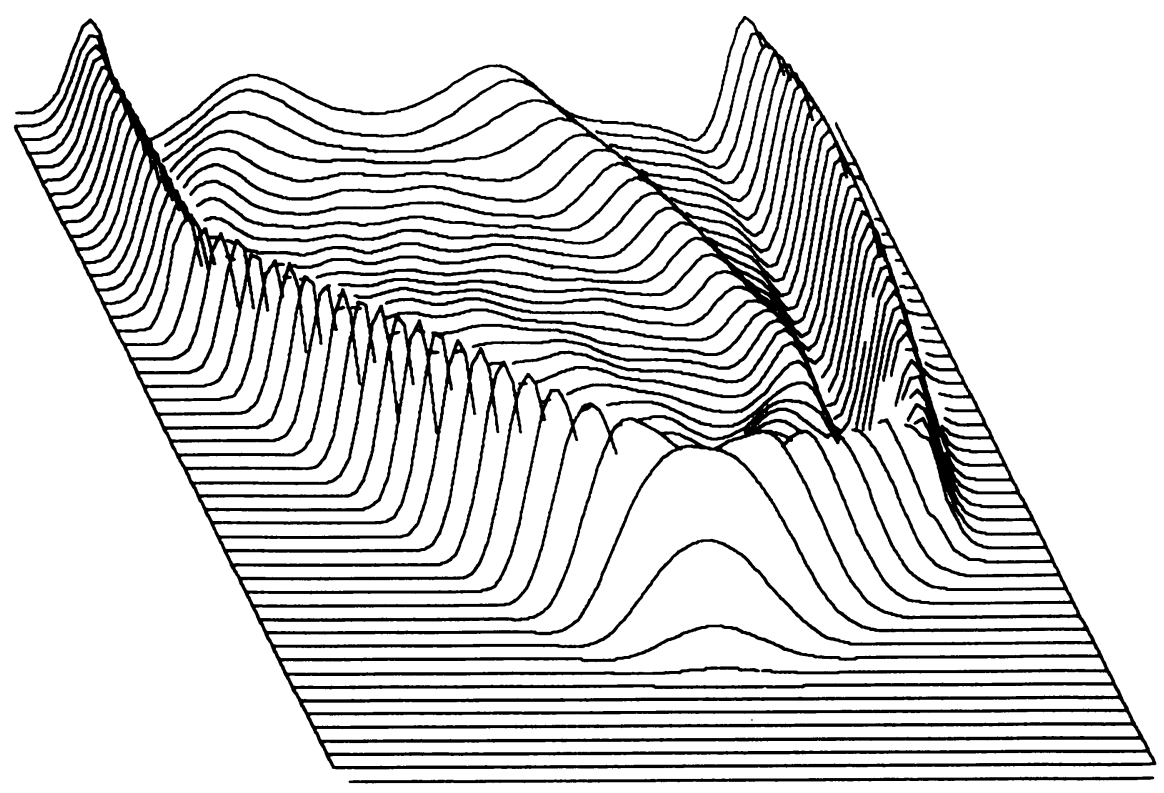

FIGURE 6b. Fourth-order method $t=.8$

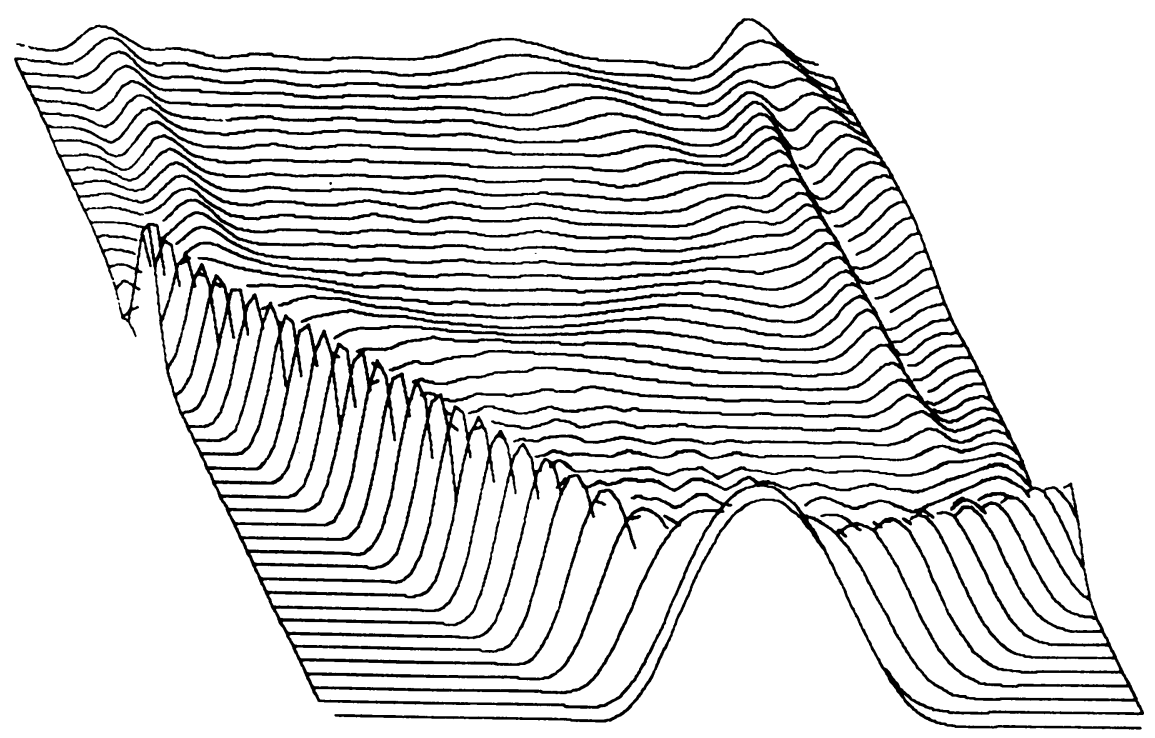

FIGURE 6c. Fourth-order method $t=1.2$ 


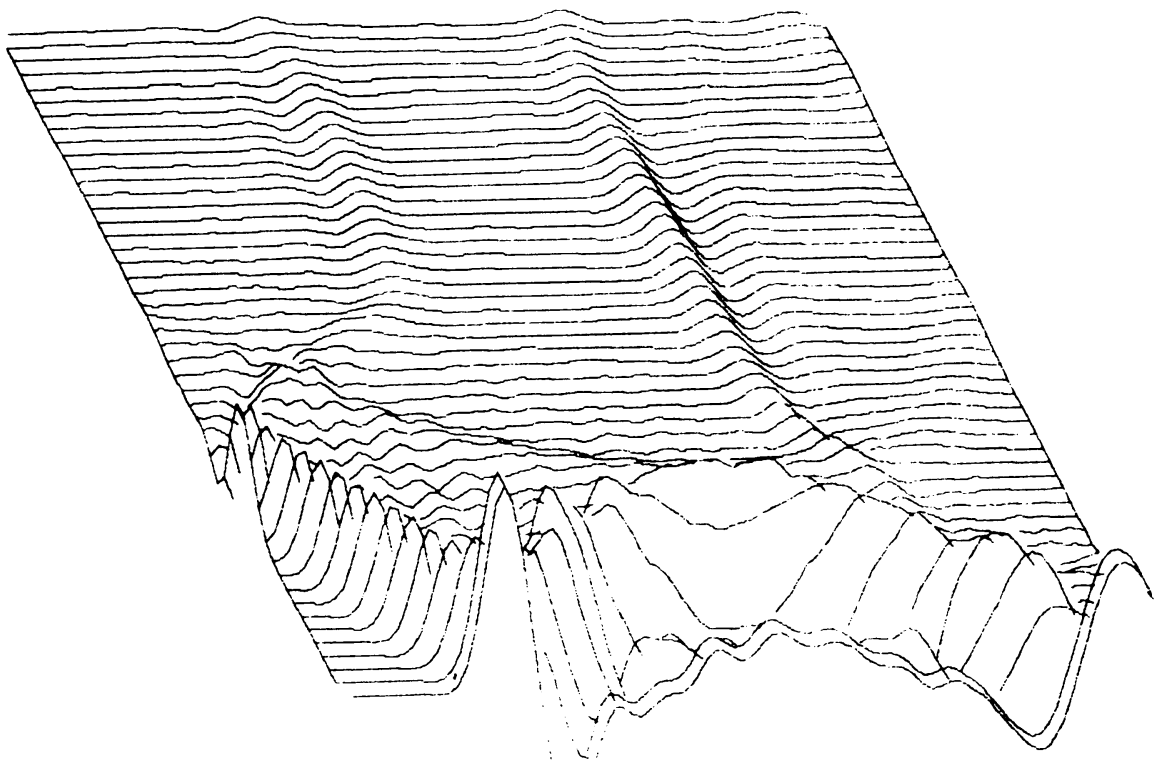

FIGURE 6d. Fourth-order method $t=1.6$

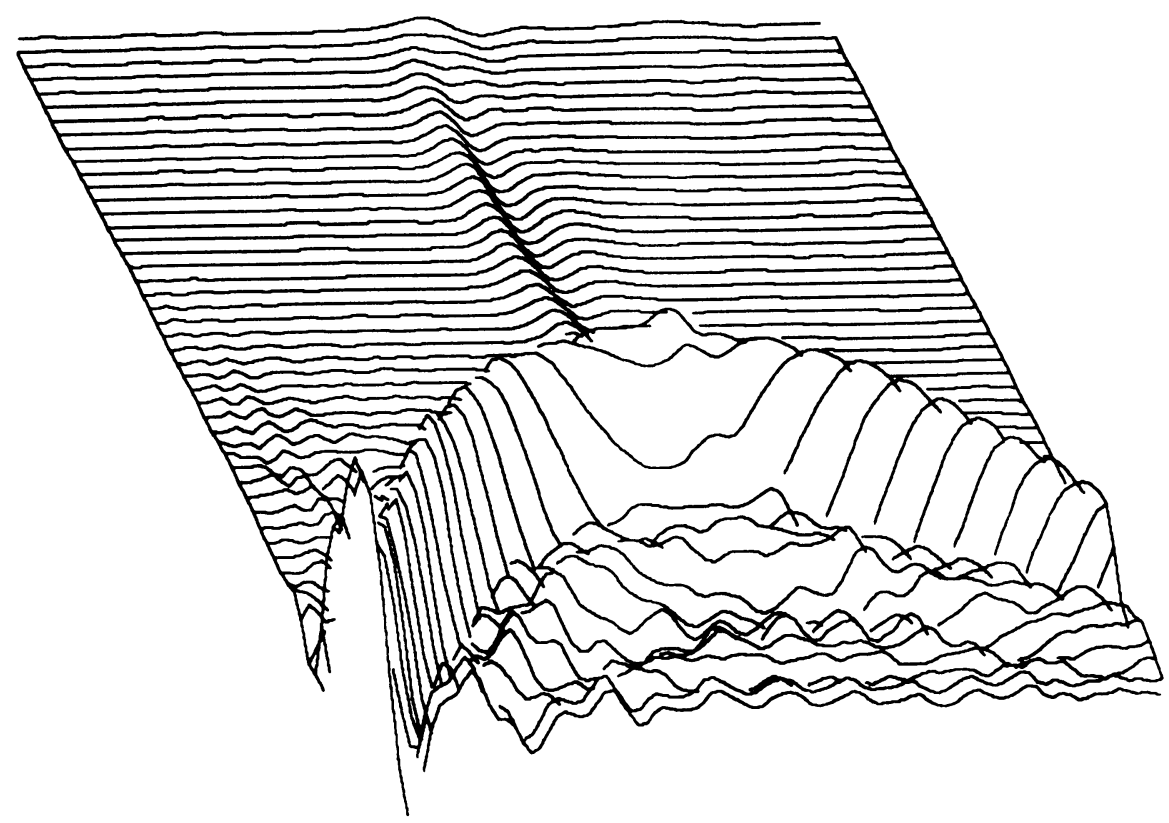

FIGURE 6e. Fourth-order method $t=2.0$ 


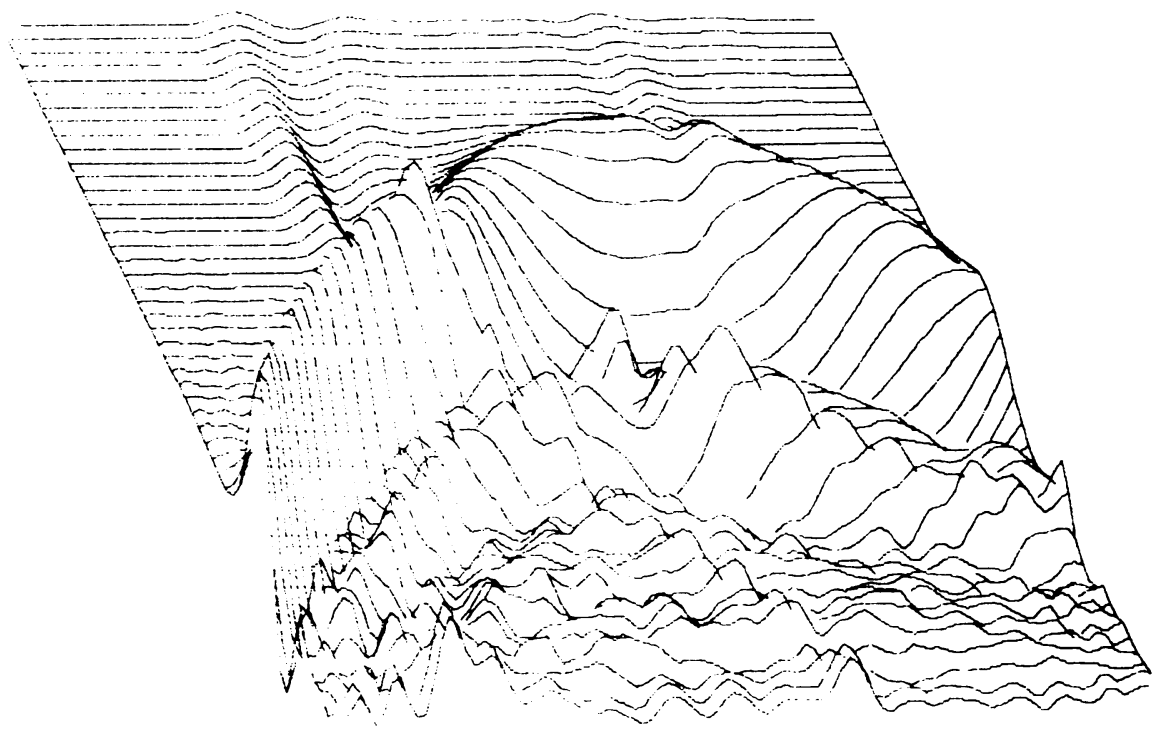

FIGURE 6f. Fourth-order method $t=2.4$

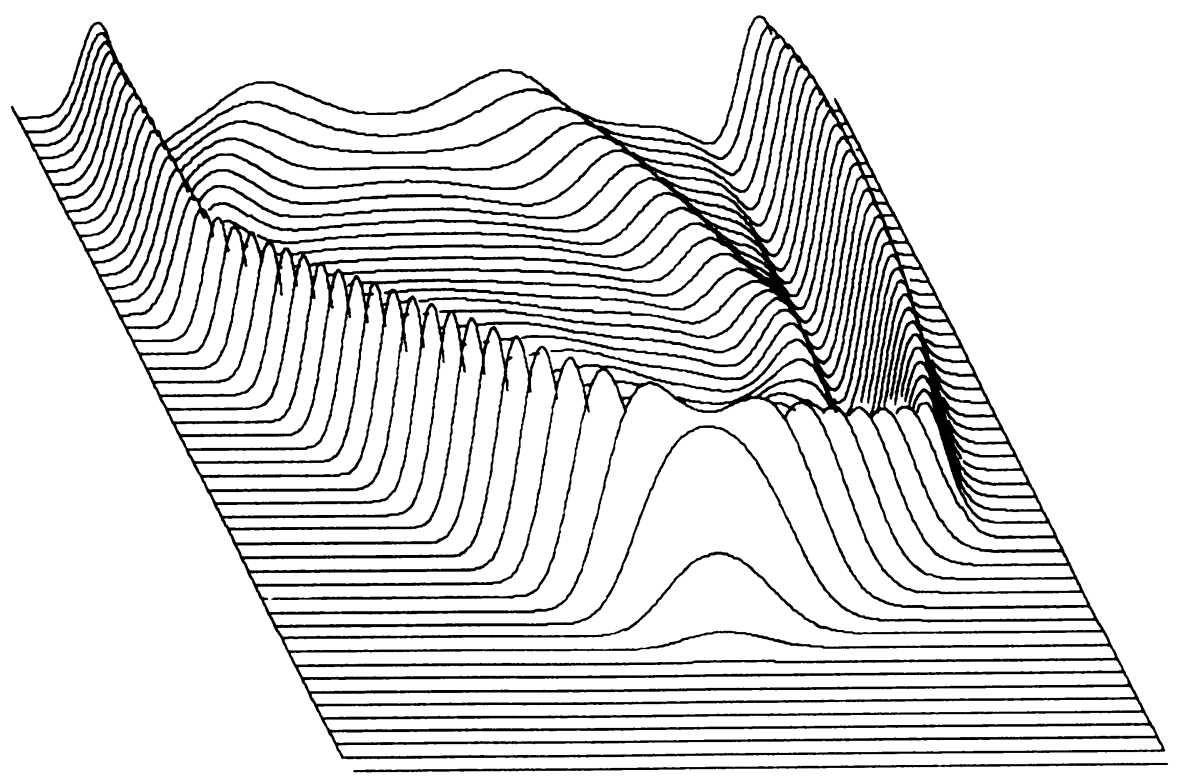

FIGURE 7a. Fourth-order method, fine mesh $t=.8$ 


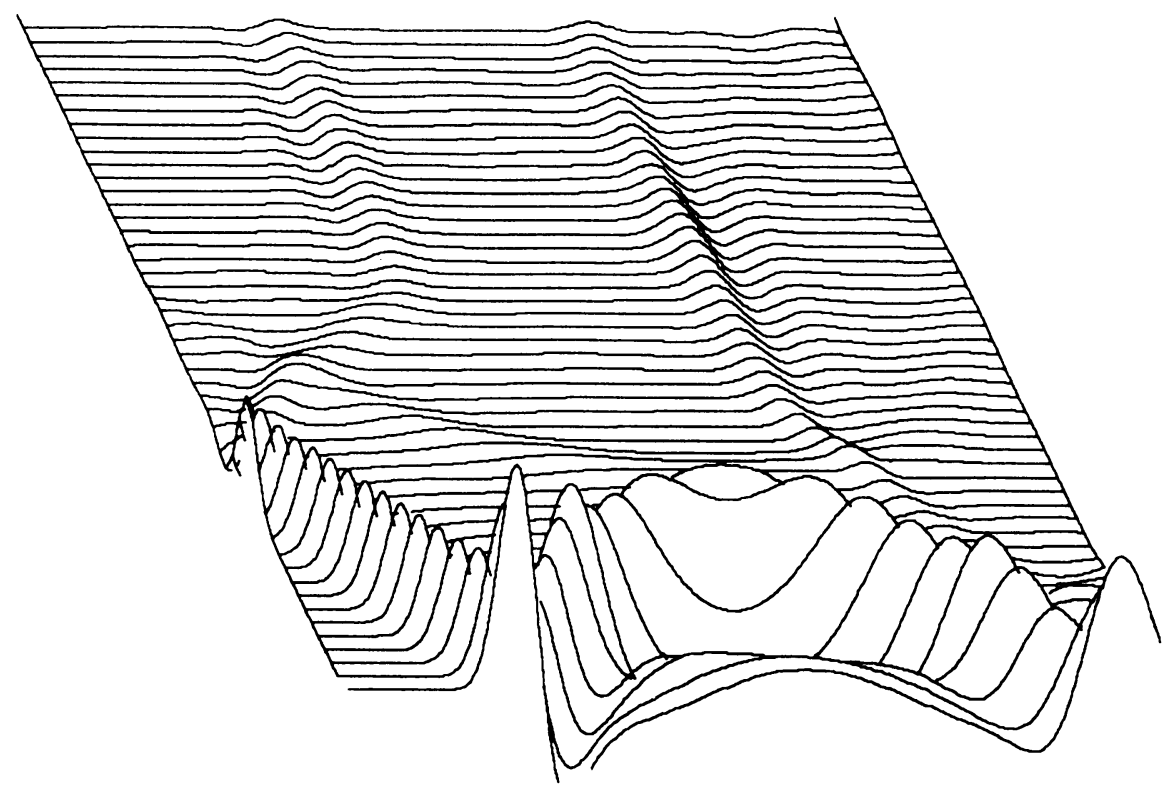

FIGURE 7b. Fourth-order method, fine mesh $t=1.6$

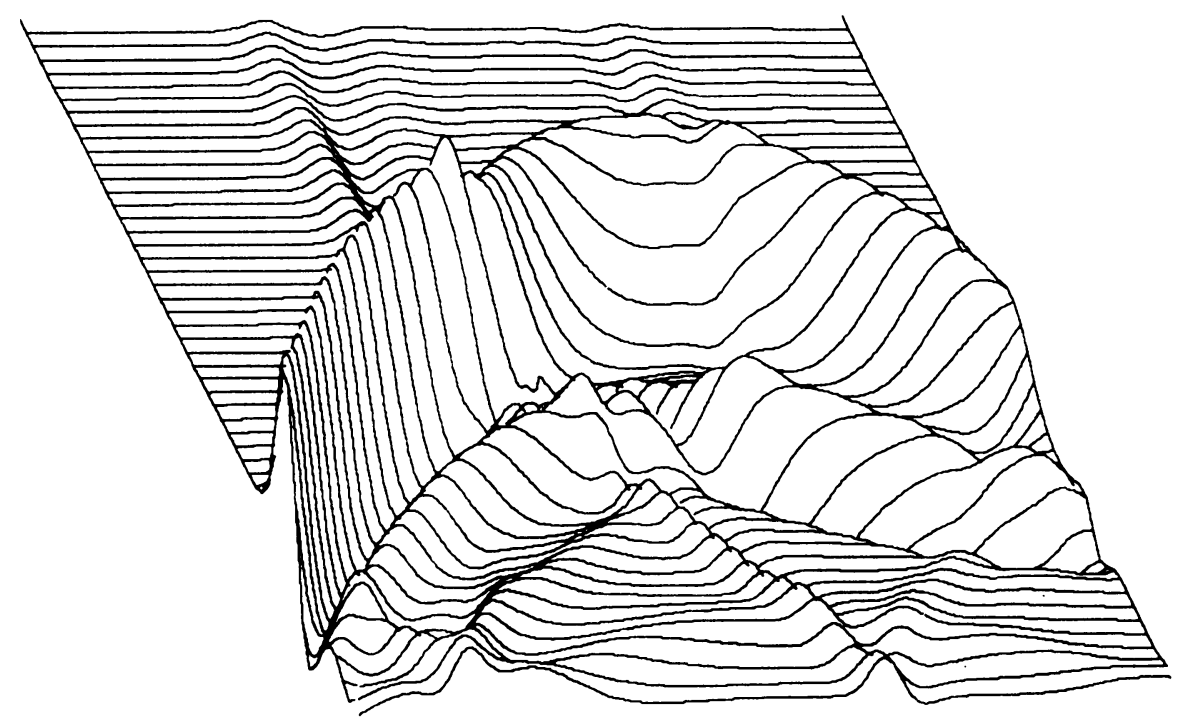

FIGURE 7c. Fourth-order method, fine mesh $t=2.4$ 
mesh. We approximate the wave equation in two space dimensions,

$$
u_{t t}=\left(c^{2} u_{x}\right)_{x}+\left(c^{2} u_{y}\right)_{y}
$$

with a second-order method,

$$
\begin{aligned}
D_{+, t} D_{-, t} w(x, y, t)= & D_{+, x}\left(c^{2}\left(x-\frac{1}{2} h, y, t\right) D_{-, x} w(x, y, t)\right) \\
& +D_{+, y}\left(c^{2}\left(x, y-\frac{1}{2} h, t\right) D_{-. y} w(x, y, t)\right),
\end{aligned}
$$

and a fourth-order method in which the term

$$
\begin{aligned}
& \frac{k^{2} h^{2}}{24}\left(D_{+, x}\left(c^{2}\left(x-\frac{1}{2} h, y, t\right) D_{+, x} D_{-, x}^{2} w(x, y, t)\right)\right. \\
& \left.\quad+D_{+, x}^{2} D_{-, x}\left(c^{2}\left(x-\frac{1}{2} h, y, t\right) D_{-, x} w(x, y, t)\right)\right)
\end{aligned}
$$

and a similar term in $y$ are subtracted from the right-hand side of (3.17). (Here the notations $D_{+, q}$ and $D_{-, q}$ are used to denote the forward and backward divided differences in the $q$-direction.) The computational region is given by $0 \leqslant x \leqslant 1$, $0 \leqslant y \leqslant 2$. The wave speed $c$ is given by

$$
c(x)= \begin{cases}.5 & \text { for } x<.3+y / 5 \\ 1.0 & \text { for } x>.3+y / 5\end{cases}
$$

and the initial conditions for the difference approximation are given by

$$
w(x, y, 0)=w(x, y, k)=\exp \left(-200\left((x-1 / 5)^{2}+(y-1)^{2}\right)\right),
$$

which models a circularly symmetric source that is initially moving both inwards and outwards with respect to its center. The boundary conditions were chosen to model transparent boundaries at $x=0, y=0$ and $y=2$ and a reflective boundary at $x=1$. The actual conditions used were difference approximations to the "absorbing" type A1 boundary condition of Clayton and Engquist [ 2, p. 1531] for the first three conditions and a numerical approximation to $u_{x}(1, y, t)=0$ for the final boundary condition. For all computations, the mesh was uniform in both $x$ and $y$ and the timestep ratio used was $k / h=.5$, where $h=\Delta x=\Delta y$ is the mesh width in both the $x$ and $y$ directions. Figures $5 \mathrm{a}-\mathrm{g}$ show the numerical solution of this problem computed using the second-order method (3.17). The solution is displayed in hidden-line plots for uniformly spaced times between $t=0$ and $t=2.4$. Fifty points in $x$ and 100 points in $y$ were used in the computational mesh. Figures $6 \mathrm{a}-\mathrm{f}$ show the numerical solution of the problem computed using the fourth-order method. Note that even after the waves interact with the interface, the fourth-order method gives much "cleaner" results. It is particularly evident in the plots for $t=1.6$ and later that the dispersion error is significantly larger for the second-order method than for the fourth-order method. For comparison, the same computation was made with the fourth-order method on a finer mesh (150 points in $x$ and 300 points in $y$ ). These results are shown in Figures 7a-c. Comparison of the various plots for $t=2.4$ indicate that some of the lower amplitude waves in the solution are much more readily discernable in the fourth-order coarse mesh computations than in the corresponding second-order results. These computations indicate that the analysis for the one-dimensional case given in Section 2 gives a good picture of what to expect in two-dimensional computations as well. It is clear that the numerical group 
velocity is better approximated in the fourth-order example than in the second-order example, even after the interaction with the interface takes place. This again verifies the main point of this note, which is to point out that if one is interested in obtaining qualitatively correct behavior in linear wave propagation problems, the accuracy with which the phase or group velocity is approximated is more important than the accuracy with which internal boundary conditions are represented.

Acknowledgements. I am grateful to Professors H. O. Kreiss and Tim Minzoni for helpful discussions and also to Dr. L. N. Trefethen for rekindling my interest in this problem.

Department of Applied Mathematics

California Institute of Technology

Pasadena, California 91125

1. Z. Alterman \& F. C. Karal, JR., "Propagation of elastic waves in a layered media by finite difference methods”, Bull. Seismol. Soc. Amer., v. 58, 1968, pp. 367-398.

2. R. W. Clayton \& B. ENGQuist, “Absorbing boundary conditions for acoustic and elastic wave equations,” Bull. Seismol. Soc. Amer., v. 67, 1977, pp. 1529-1540.

3. B. GuSTAFSSON, H. O. KREISS \& A. SUnDSTRÖM, "Stability theory of difference approximations for mixed initial boundary problems. II,” Math. Comp., v. 26, 1972, pp. 649-686.

4. K. R. Kelly, R. W. Ward, Sven Treitel \& R. M. Alford, "Synthetic seismograms; A finite-difference approach." Geophysics, v. 41, 1976, pp. 2-27.

5. A. N. Tiknonov \& A. A. SamarskiI, "Homogeneous difference schemes," Zh. Vychisl. Mat. i Mat. Fiz., v. 1, 1961, pp. 5-63.

6. L. N. Trefethen, “Group velocity in finite difference schemes," SIAM Rev'., v. 24, 1982, pp. $113-136$. 